\title{
Glycine-alanine dipeptide repeat protein contributes to toxicity in a zebrafish model of C9orf72 associated neurodegeneration
}

\author{
Yu Ohki ${ }^{1,2}$, Andrea Wenninger-Weinzierl ${ }^{1}$, Alexander Hruscha ${ }^{1}$, Kazuhide Asakawa ${ }^{3}$, Koichi Kawakami ${ }^{3}$ \\ Christian Haass ${ }^{1,2,4}$, Dieter Edbauer ${ }^{1,4}$ and Bettina Schmid ${ }^{1,4^{*}}$
}

\begin{abstract}
Background: The most frequent genetic cause of frontotemporal lobar degeneration (FTLD) and amyotrophic lateral sclerosis (ALS) is the expansion of a GGGGCC hexanucleotide repeat in a non-coding region of the chromosome 9 open reading frame 72 (C9orf72) locus. The pathological hallmarks observed in C9orf72 repeat expansion carriers are the formation of RNA foci and deposition of dipeptide repeat (DPR) proteins derived from repeat associated non-ATG (RAN) translation. Currently, it is unclear whether formation of RNA foci, DPR translation products, or partial loss of C9orf72 predominantly drive neurotoxicity in vivo. By using a transgenic approach in zebrafish we address if the most frequently found DPR in human ALS/FTLD brain, the poly-Gly-Ala (poly-GA) protein, is toxic in vivo.

Method: We generated several transgenic UAS responder lines that express either 80 repeats of GGGGCC alone, or together with a translation initiation ATG codon forcing the translation of GA80-GFP protein upon crossing to a Gal4 driver. The GGGGCC repeat and GA80 were fused to green fluorescent protein (GFP) lacking a start codon to monitor protein translation by GFP fluorescence.

Results: Zebrafish transgenic for the GGGGCC repeat lacking an ATG codon showed very mild toxicity in the absence of poly-GA. However, strong toxicity was induced upon ATG initiated expression of poly-GA, which was rescued by injection of an antisense morpholino interfering with start codon dependent poly-GA translation. This morpholino only interferes with GA80-GFP translation without affecting repeat transcription, indicating that the toxicity is derived from GA80-GFP.

Conclusion: These novel transgenic C9orf72 associated repeat zebrafish models demonstrate poly-GA toxicity in zebrafish. Reduction of poly-GA protein rescues toxicity validating this therapeutic approach to treat C9orf72 repeat expansion carriers. These novel animal models provide a valuable tool for drug discovery to reduce DPR associated toxicity in ALS/FTLD patients with C9orf72 repeat expansions.
\end{abstract}

Keywords: Zebrafish, C9orf72, poly-GA toxicity

\section{Background}

Expansion of the GGGGCC hexanucleotide repeat in the C9orf72 intronic region was recently identified as a cause for amyotrophic lateral sclerosis (ALS) and frontotemporal lobar degeneration (FTLD) [1-3]. This repeat expansion is observed in around $40 \%$ of familial and $7 \%$ of sporadic cases of ALS and $25 \%$ of familial and $6 \%$ of

\footnotetext{
* Correspondence: bettina.schmid@dzne.de

${ }^{1}$ German Center for Neurodegenerative Diseases (DZNE),

Feodor-Lynen-Str.17, 81377 Munich, Germany

${ }^{4}$ Munich Cluster for Systems Neurology (SyNergy), Feodor-Lynen-Str.17,

81377 Munich, Germany

Full list of author information is available at the end of the article
}

sporadic cases of FTLD [4]. Affected patients have hundreds to several thousands of repeats, while healthy individuals generally have 2 to 23 repeats $[1-3,5]$. The expanded repeat RNA is transcribed and accumulates in RNA foci, which have been detected in brain tissue, lymphoblasts, as well as fibroblasts derived from patients with the C9orf72 associated repeat expansion [6]. This long repeat RNA transcript can sequester RNA binding proteins, including heterogeneous nuclear ribonucleoprotein A3 (hnRNPA3), hnRNPH, and nucleolin, and can lead to mis-regulation of RNA splicing [7-9]. Interestingly, despite the absence of an ATG start codon, the repeat 
RNA is further subjected to unconventional repeat associated non-ATG (RAN) translation [7, 9-11] resulting in dipeptide repeat proteins (DPRs) of Gly-Ala (poly-GA), Gly-Arg (poly-GR) and Gly-Pro (poly-GP) and additional Gly-Pro (poly-GP), Pro-Ala (poly-PA) and Pro-Arg (polyPR) from the transcribed antisense strand. The DPRs form cytosolic coaggregates with p62 in the brains of patients with C9orf72 repeat expansions [12-15] and have been shown to interfere with RNA metabolism and coaggregate with other proteins [16-20]. Additionally, interference of DPRs with nucleocytoplasmic transport has been identified independently in different model systems by unbiased genetic screens [21-25].

Three pathomechanisms have been postulated in C9orf72 repeat expansion carriers, which are not mutually exclusive and most likely act in combination. First, haploinsufficiency due to reduced transcript levels of C9orf72. Second, toxicity of RNA foci by sequestration of important RNA binding proteins and disturbed RNA homeostasis. Third, toxicity of RAN translation products.

We set out to generate transgenic zebrafish lines with expanded GGGGCC repeats and poly-GA as a vertebrate animal model to address their contribution to toxicity. We generated two transgenic lines expressing 80 repeats of the GGGGCC sequence (ggggcc80) and two lines with the translation initiation codon ATG in front of the 80xGGGGCC repeat sequence driving expression of poly-GA protein fused to green fluorescent protein (GA80-GFP). We chose poly-GA since it is the most abundant DPR species found in patients with C9orf72 repeat expansions [13, 26]. The transgenic zebrafish models with 80 repeats reproduced key pathological features, such as RNA foci, however RAN translation was not detectable. Transgenic zebrafish with 80 repeats of GGGGCC only showed minor toxicity (mild pericardial edema), which was greatly increased when we forced expression of poly-GA by 80 GGGGCC repeats with an ATG translational start codon in the GA frame (severe pericardial edema). By blocking poly-GA translation by an antisense approach, we show that the phenotypes can be partially rescued, demonstrating that poly-GA is toxic in vivo and that targeting poly-DPRs might be a useful therapeutic strategy for C9orf72 repeat expansion carriers.

\section{Results}

Generation of a transgenic zebrafish model of C9orf72 repeat expansion disease

We generated several transgenic zebrafish UAS-based responder lines expressing either 2 or 80 repeats with an ATG (Tg(UAS:ATG-2xGGGGCC-GFP) and Tg(UAS:ATG80xGGGGCC-GFP)) in the GA reading frame as well as 80 GGGGGCC repeats without ATG (Tg(UAS:80xGGGGCCGFP)) fused to GFP (Fig. 1a). We generated these lines by
Tol2 mediated transposition into the zebrafish genome [27]. We confirmed successful germline transmission of the transgenes by PCR-based genotyping. The 80xGGGGCC repeat sequence was unstable and changes in repeat length were frequently observed in the F1 generation (data not shown). We selected stable transgenic lines with 80xGGGGCC repeats and confirmed the repeat length by PCR (Fig. 1b). To exclude potential toxicity mediated by the transgene integration site, we selected 2 independent lines ( $\mathrm{a}$ and $\mathrm{b}$ ) for $\mathrm{Tg}$ (UAS:ATG-2xGGGGCC-GFP) and $\mathrm{Tg}$ (UAS:ATG-80xGGGGCC-GFP), which we will refer to as GA2-GFPa/b and GA80-GFPa/b respectively. Comparable mRNA expression levels of the respective transgene were confirmed by semi-quantitative reverse transcription PCR (RT-PCR) upon crossing the responder lines to the gene trap Gal4 driver line SAGFF73A [28]. This line drives expression of high level of Gal4 ubiquitously at early embryonic stages and was used in all experiments to drive transgene expression of the respective UAS driver lines. All transgenic lines showed comparable RNA expression levels at 4 days post fertilization (dpf) (Fig. 1c). Additionally, we confirmed the expression of GA80-GFP protein by immunoblotting at $4 \mathrm{dpf}$ (Fig. 1d). The GA2-GFPa/b larvae showed 7.2 fold higher GFP protein expression levels compared to the GA80-GFPa/b larvae (mean of line $a$ and $b$ ). This suggests that despite nearly equal mRNA levels, less poly-GA is translated in the GA80-GFP fish than the GA2GFP fish. A poly-GA specific antibody also detected the GA80-GFP protein (Fig. 1d), but failed to detect GA2-GFP, most likely because two GA repeats are too short to be recognized by the antibody.

$\mathrm{Tg}$ (UAS:80xGGGGCC-GFP) fish expressing repeat RNA without a start codon were generated and fish with a stable length of 80 repeats were selected by PCR (Fig. 1c). This line will be referred to as ggggcc80-GFP. Although the expression level of mRNA is similar to those of GA2-GFP and GA80-GFP expressing fish, polyGA peptides derived from RAN translation were not detectable by Western blotting in the ggggcc80-GFP fish at $4 \mathrm{dpf}$ (Fig. 1d). We also failed to detect poly-GR and poly-GP (data not shown). Thus, if RAN translation occurs in the ggggcc80-GFP line, it is below the detection limit of our specific antibodies.

\section{RNA derived from GA80-GFP and ggggcc80-GFP lead to RNA foci formation}

To test if pathological hallmarks of C9orf 72 repeat expansion disease are found in transgenic zebrafish with an expanded GGGGCC repeat, we analyzed RNA foci formation at $28 \mathrm{~h}$ post fertilization (hpf). GA80-GFP as well as ggggcc80-GFP zebrafish showed RNA foci in spinal cord neurons by in situ hybridization, whereas wild-type and GA2-GFP zebrafish did not show RNA foci (Fig. 2a and Additional file 1: Figure S1). We further 
A
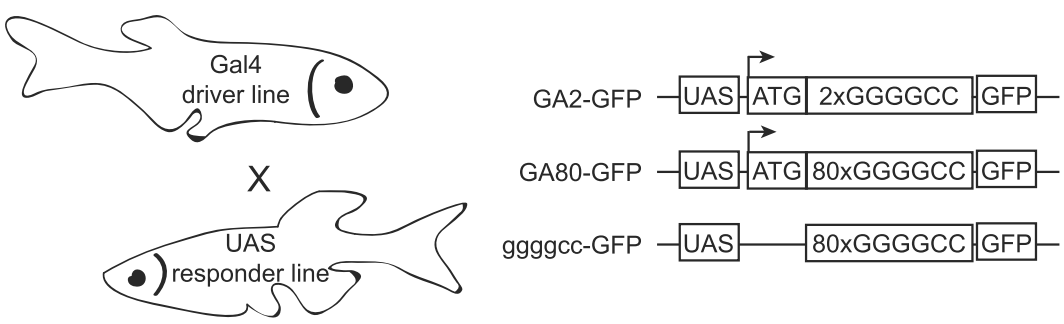

ggggcc-GFP UAS $80 \times$ GGGGCC GFP-

B

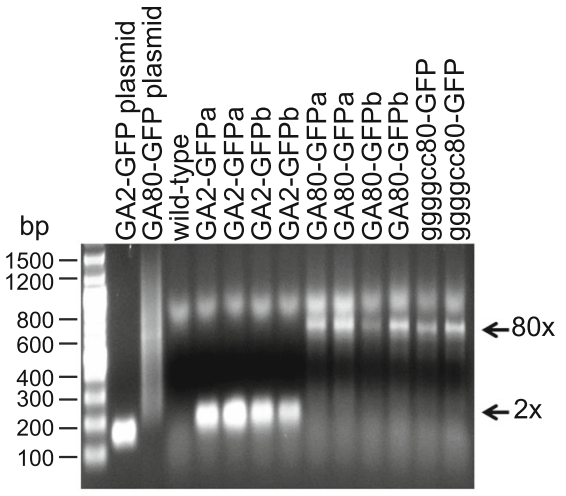

C

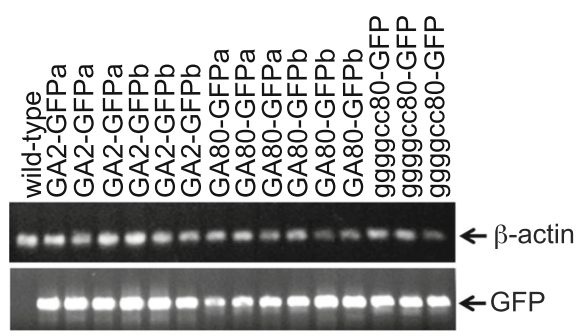

D

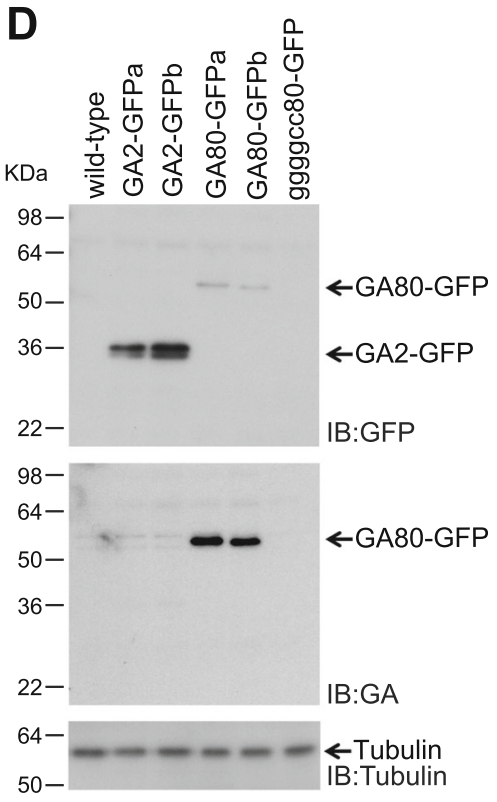

Fig. 1 Generation of transgenic zebrafish model of C9ORF72 repeat expansion disease. a Schematic representation of Gal4 driver line zebrafish crossed to a UAS responder transgenic zebrafish to generate embryos that express a transgene under the control of the UAS. Schematic representation of the responder constructs used for the generation of transgenic zebrafish. $\mathbf{b}$ Genotyping by PCR of 1 dpf embryos. pCS2 + 2xGGGGGCC and pCS2 + 80XGGGGCC constructs were used as standards for GA2-GFP and GA80-GFP in lane 2 and 3. Positions of 2xGGGGCC and 80xGGGGCC repeats are indicated by arrows. c Semi-quantitative RT-PCR analyses for the wild-type, GA2-GFPa/b, GA80-GFPa/b and ggggcc80-GFP zebrafish. Note that all transgenic lines showed similar expression level at 4 dpf embryos. $\mathbf{d}$ Immunoblotting of wild-type, GA2-GFPa/b, GA80-GFPa/b and ggggcc80-GFP with antibodies as indicated with embryonic lysates of 4 dpf old embryos

confirmed that the RNA foci are sensitive to RNaseA but not to DNase treatment, confirming the RNA nature of the foci (Fig. 2b and Additional file 1 Figure S1).

\section{ggggec80-GFP expression displays mild toxicity in zebrafish}

We next analyzed ggggcc80-GFP transgenic fish for signs of toxicity. ggggcc80-GFP larvae showed a pericardial edema phenotype at $4 \mathrm{dpf}$. We categorized the phenotypes based on their severity in three groups: 1. wild-type, 2 . mild edema and 3. severe pericardial edema as shown in Fig. 2c. Of the ggggcc80-GFP larvae $50.2 \pm 13.7 \%$ (mean \pm SD) were unaffected, $32.2 \pm 8.2 \%$ had a mild pericardial edema and $17.2 \pm 11.6 \%$ had a severe pericardial edema at $4 \mathrm{dpf}$ (Fig. $2 \mathrm{~d}$ ). The edema phenotype precluded inflation of the swim bladder, inability to feed independently, and death during early larval stages. No abnormal phenotype was detectable in GA2-GFP larvae, suggesting that the expanded GGGGCC repeat causes the mild toxicity in the ggggcc80-GFP fish.

\section{GA80-GFP protein expression is highly toxic in zebrafish}

Poly-GA has previously been shown to be toxic in neurons and animal models [23, 29]. Expression of GA80-GFP protein was detected by diffuse green GFP expression and as GFP inclusions exclusively in the musculature by 2 and $4 \mathrm{dpf}$ (Fig. 3a), whereas GA2-GFP fish showed only diffuse green fluorescence. Almost all GA80-GFPa expressing fish showed a severe pericardial edema $(92.8 \pm 2.8 \%)$ and only very few fish showed a mild pericardial edema $(7.2 \pm 2.8 \%)$ at $4 \mathrm{dpf}$. Similarly, the second transgenic line GA80-GFP-b had $97.5 \pm 2.6 \%$ 


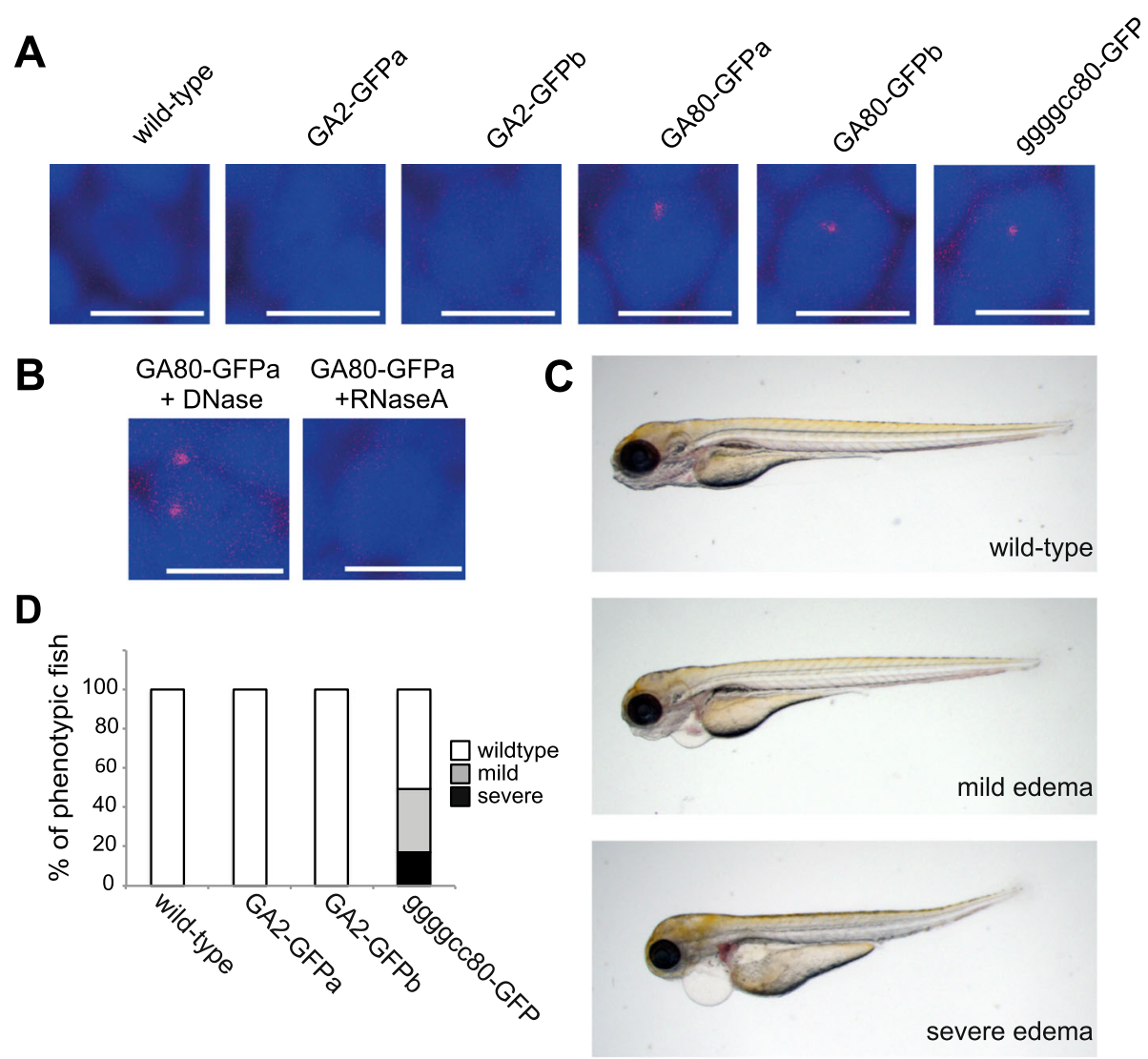

Fig. 2 RNA foci formation in transgenic zebrafish. a, b Cy3-labeled in situ probe detected dot-like structures in spinal cord in GA80-GFPa/b and ggggcc80-GFP zebrafish. b Foci were only detected in GA80-GFP and ggggcc-GFP fish whereas no foci were detected in wild-type and GA2-GFPa/b fish at 28 hpf. GA80-GFPa zebrafish were treated with RNaseA or DNase. Scale bar $10 \mu \mathrm{m}$. c Pericardial edema phenotype observed in ggggcc80-GFP zebrafish at $4 \mathrm{dpf}$. Phenotypic features are classified as wild-type, mild edema, and severe edema. $\mathbf{d}$ The average percentages of phenotypic fish of the three different phenotypic classes at $4 \mathrm{dpf}$ are indicated in the bargraph (at least three independent clutches were analyzed with $n \geq 14$ )

of the fish with an severe pericardial edema and $2.5 \pm$ $2.6 \%$ with an mild pericardial edema at $4 \mathrm{dpf}$ (Fig. 3b).

Additionally, GA80-GFPa/b fish also showed a strongly reduced circulation of red blood cells in $61.5 \pm 9.0 \%$ (line a) and $21.6 \pm 3.9 \%$ (line b) of the GFP positive embryos at $2 \mathrm{dpf}$, respectively. In contrast, wild-type, GA2-GFPa/b as well as ggggcc80-GFP fish did not show any circulation defect of red blood cells at $2 \mathrm{dpf}$ (Fig. 3c,d), suggesting that poly-GA toxicity additionally impaired circulation. The circulation defect did not seem to be mediated by a heart defect since the GA80-GFPa fish had a normal heart beat at $2.5 \mathrm{dpf}$ (data not shown). Phenotypic embryos with a strong edema and lack of circulation died around $5 \mathrm{dpf}$.

We further examined the length of the spinal motor neuron axons in GA80-GFPa zebrafish, since C9orf72 repeat expansion carriers can suffer from motor neuron degeneration. No significant differences of the axonal length were observed at $28 \mathrm{hpf}$ (Additional file 2: Figure S2A, B). In addition, there was no apparent difference in the overall neuronal outgrowth and branching at $2 \mathrm{dpf}$
(Additional file 3: Figure S3A, B), indicating expression of neither an expanded GGGGCC repeat nor poly-GA protein affected neuronal outgrowth. Interestingly, aggregates of GA80-GFP were exclusively found in the musculature despite ubiquitous GA80-GFP protein expression in both transgenic GA80-GFP lines at 2 and 4 dpf. Larvae with a severe edema phenotype had more than twice as many inclusions compared to larvae with a mild phenotype at $4 \mathrm{dpf}$ (Additional file 4: Figure S4A), suggesting that these embryos have higher expression levels, leading to higher toxicity and more inclusions. The overall structure of the muscle was not affected as determined by $\alpha$-actinin staining at $2 \mathrm{dpf}$ (Additional file 4: Figure S4B), suggesting that the GA80-GFP aggregates themselves are not toxic and that muscle defects are not the primary cause of toxicity in our zebrafish model.

Previously, we reported that knockout of the two orthologues of human TDP-43, a key protein in ALS/ FTLD, in zebrafish (tardbp-/-, tardbpl-/-) showed a circulation phenotype accompanied with mispatterning 

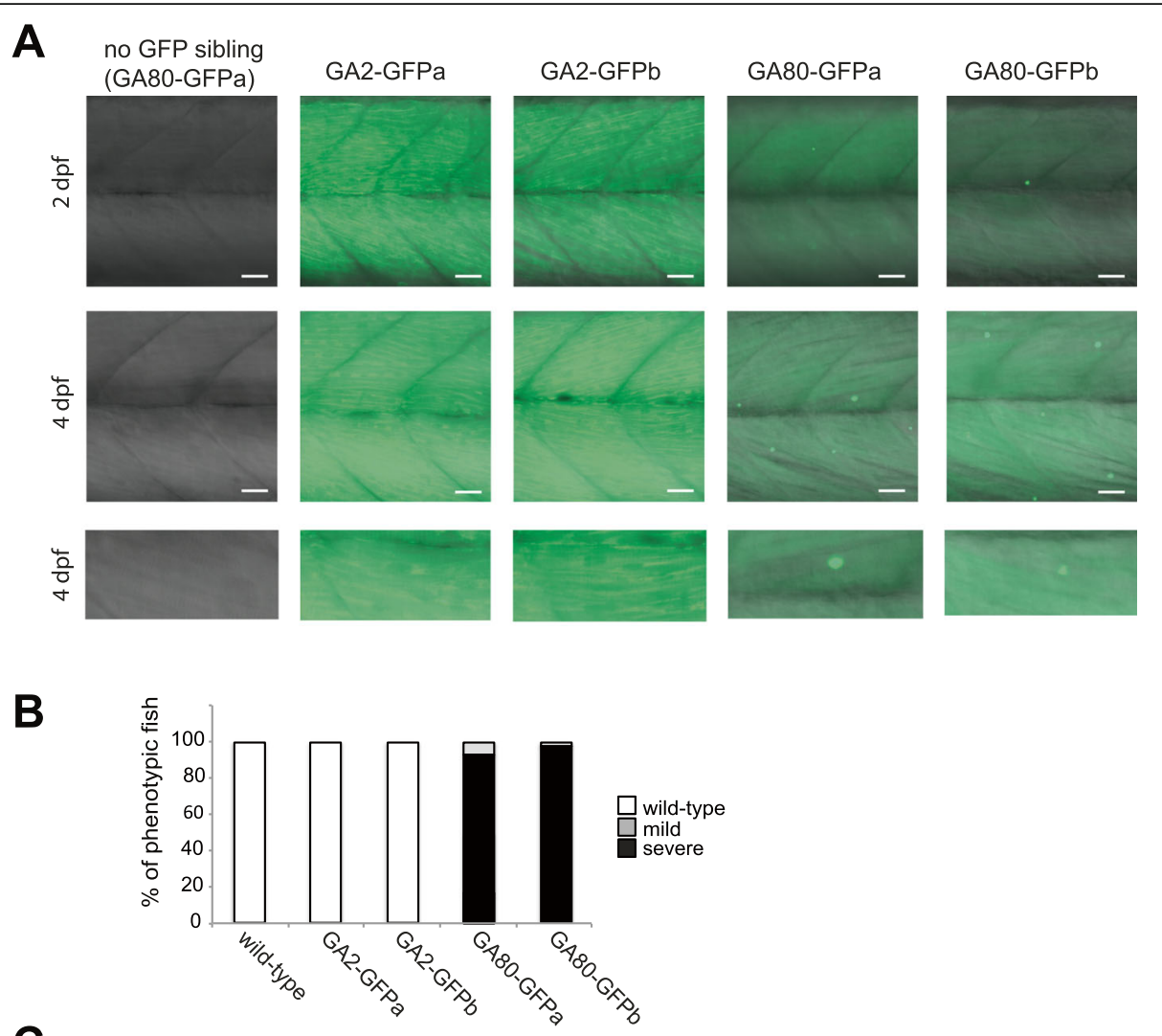

C

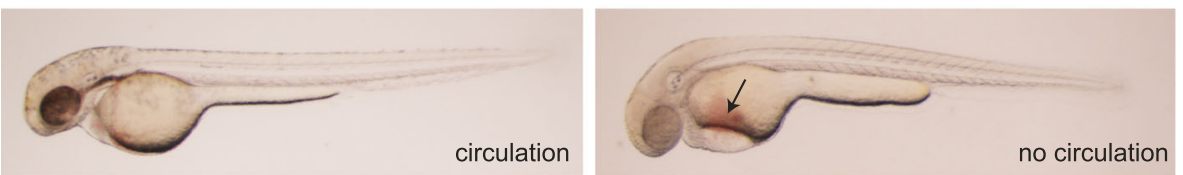

D

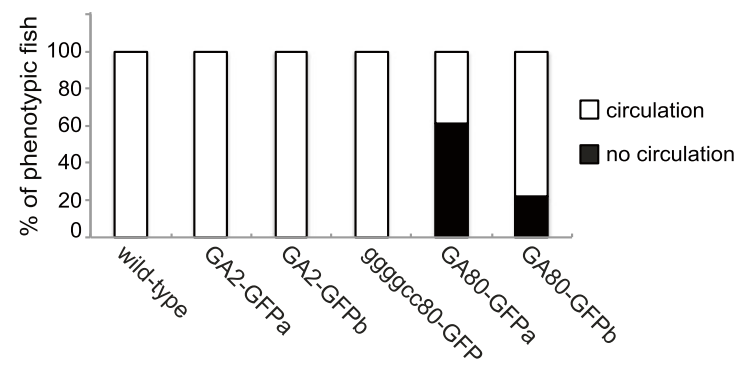

Fig. 3 Poly-GA protein elicits a toxic phenotype. a In vivo image of GA-GFP polypeptides at 2 and 4 dpf. Genotypes as indicated. No GFP sibling (GA80-GFP) refers to a sibling from a cross between a Gal4 driver and a UAS GA80-GFP responder fish that is GFP negative, and hence is either negative for the driver or the responder construct, or both constructs. GFP fluorescent images shown are merged with DIC pictures. Lowest panel is a magnification of the middle panel at $4 \mathrm{dpf}$. Lateral views of the trunk musculature. Scale bar $20 \mu \mathrm{m}$. b A strong pericardial edema phenotype was observed in GA80-GFPa/b zebrafish at $4 \mathrm{dpf}$. The average percentages of phenotypic fish of the three different classes are indicated in the bargraph.

c GA80-GFPa/b zebrafish had mostly no circulation at $2 \mathrm{dpf}$. Red blood cells accumulate due to circulation defects (arrow). d The average percentages of fish with or without circulation are indicated in the bargraph (at least three independent clutches were analyzed with $n \geq 14$ )

and increased sprout formation of the vasculature [19]. Since C9orf72 repeat expansion carriers show cytoplasmic TDP-43 mislocalization and presumably partial TDP-43 loss of function, we analyzed TDP-43 function in the repeat expressing fish. We examined vascular patterning in GA80-GFPa zebrafish by crossing them to
$\mathrm{Tg}(\mathrm{kdrl} \text { :HsHRAS-mCherry })^{\mathrm{s} 896}$, a reporter line expressing mCherry in all endothelial cells [20]. However, neither vascular mispatterning nor aberrant sprout formation as seen in $\operatorname{tardbp-/-,~tardbpl-/-~fish~were~}$ detected at $2.5 \mathrm{dpf}$ (Additional file 5: Figure S5A, B), ruling out vascular patterning defects as the primary cause 
of the circulation phenotype. However, endothelial cells appeared thinner and less structured in GA80-GFPa larvae (Additional file 5: Figure S5), most likely due to the lack of perfusion. Reduction of zebrafish Tardbp function leads to an alternative splicing pattern of the second ortholgue Tardbpl, referred to as Tardbpl_tv1, which can fully replace Tardbp function. Impaired Tardbp function (for example by mislocalization) can therefore be monitored by upregulation of the compensatory Tardbpl_tv1 variant. We analyzed the expression levels of Tardbp as well as Tardbpl_tv1 by immunoblotting in GA80-GFPa zebrafish at $2 \mathrm{dpf}$. Increased levels of Tardbpl_tv1 would indicate partial loss of Tardbp. However, protein levels of Tardbp and Tardbpl_tv1 showed no differences in wild-type and GA80-GFP transgenic fish (Additional file 6: Figure S6), suggesting that the perfusion phenotype is mediated by a TDP-43 independent mechanism.

\section{Antisense morpholino (AMO) rescues the edema phenotype in GA80-GFP zebrafish}

Antisense morpholinos (AMO) are a useful tool to suppress the translation of genes of interest. Here, we designed AMOs targeting GAL4 as well as the ATG start codon of the GA80-GFP transgene (Fig. 4a) to further substantiate the correlation of GA80-GFP protein levels and toxicity. Injection of the AMO targeting GAL4 into the fertilized embryos in GA80-GFPa efficiently blocked translation of the transcriptional activator Gal4 and thereby efficiently reduced GA80GFP protein expression at $2 \mathrm{dpf}$ (Fig. 4b). Although control AMO injected transgenic zebrafish showed mostly the severe edema phenotype $(95.8 \pm 3.9 \%)$ and very few mild edema phenotypes (4.2 $\pm 3.9 \%)$, GAL4 AMO injected fish showed only few severe (4.8 \pm $8.2 \%)$ and mild $(8.6 \pm 8.3 \%)$ and mostly unaffected zebrafish $(86.7 \pm 8.3 \%)$, indicating that AMO mediated GAL4 inhibition rescued the pericardial edema phenotype at $4 \mathrm{dpf}$ (Fig. 4b).

To further address if toxicity is mediated by the expression of GA80-GFP protein we designed AMO targeting ATG start codon upstream of the GGGGCC repeat (ATG AMO) and aimed at reverting the phenotype by blocking translation of the poly-GA protein in the GA80-GFPa larvae. Upon injection of ATG AMO, GA80-GFP protein expression was severely reduced, as determined by immunoblot at $2 \mathrm{dpf}$ (Fig. 4c). In addition, ATG targeting AMO reduced the severe pericardial edema phenotype in GA80-GFP expressing zebrafish demonstrating that polyGA protein is a toxic species in vivo at $4 \mathrm{dpf}$ (Fig. 4c.). We next examined the expression level of mRNA as well as RNA foci formation upon ATG targeting AMO injection, since they are blocking translation without changing the mRNA. mRNA expression levels analyzed at $2 \mathrm{dpf}$ by semi-quantitative RT-PCR were unaffected in phenotypic fish upon ATG AMO injection and RNA foci were still detectable (Fig. 4d, e, Additional file 1 Figure S1) indicating that poly-GA was toxic in zebrafish and that the phenotypic rescue is mediated by reduction of GA80-GFP protein levels.

\section{Discussion}

Expansion of a GGGGCC repeat in a non-coding region of C9orf72 is the most common cause of ALS/FTLD. Recently, RAN translation from the sense and antisense GGGGCC repeat transcript was observed in C9orf72 repeat expansion carriers generating 5 different DPR species (poly-GA, -GR, -GP, -PA and -PR) [12-14, 30]. These DPRs coaggregate with p62 and form the characteristic star shaped inclusions in C9orf72 repeat expansion carriers [13].

The relative contribution of RNA and DPR toxicity is still under debate since many conflicting results have been obtained in a variety of different model systems (reviewed in [31]). The GGGGCC repeat RNA forms foci in cells, animal models and patients and has been shown to be able to induce neuronal cell death and to sequester RNA binding proteins [8, 15, 20, 32]. However, there is only a weak correlation between RNA foci and neurodegeneration in patients [33-35]. In zebrafish RNA injection of $8 x, 38 x$, and $72 x$ GGGGCC repeats has been shown to cause RNA foci and cell death by apoptosis in a repeat length dependent manner [9]. This study did not report on RAN translation products upon repeat RNA injections in zebrafish. In line with these studies we observe RNA foci in two independent transgenic ggggcc80-GFP lines and RNA toxicity. In the ggggcc80-GFP fish we were not able to detect GA, GP, and GR species, most likely due to the relatively short repeat length or inefficient or even lack of RAN translation in early zebrafish that preclude detection of DPR species by Western blotting. This is in contrast to fly and mouse models in which repeat expression leads to DPR translation in the absence of a start codon $[18,20]$. Whether the mild toxic effects seen in ggggcc80-GFP fish is due to RNA toxicity or low level DPRs remains to be determined. To further address DPR toxicity we focused on poly-GA since it is the most abundant species found in C9orf72 repeat carriers and induced the neuronal cell death in primary cultured cell model as well as in animal models [16-18, 20]. We generated several transgenic zebrafish lines and demonstrated that polyGA is toxic in zebrafish. In primary neurons poly-GA toxicity has been attributed to sequestration of Unc119 (a trafficking factor for myristylated proteins), interference with the ubiquitin proteasome system, and endoplasmic reticulum stress $[16,17]$. Recently, poly-GR and poly-PR were shown to be the most toxic DPR species in Drosophila [18, 36]. Moreover, the arginine-rich DPR species are also toxic in primary neurons, potentially 

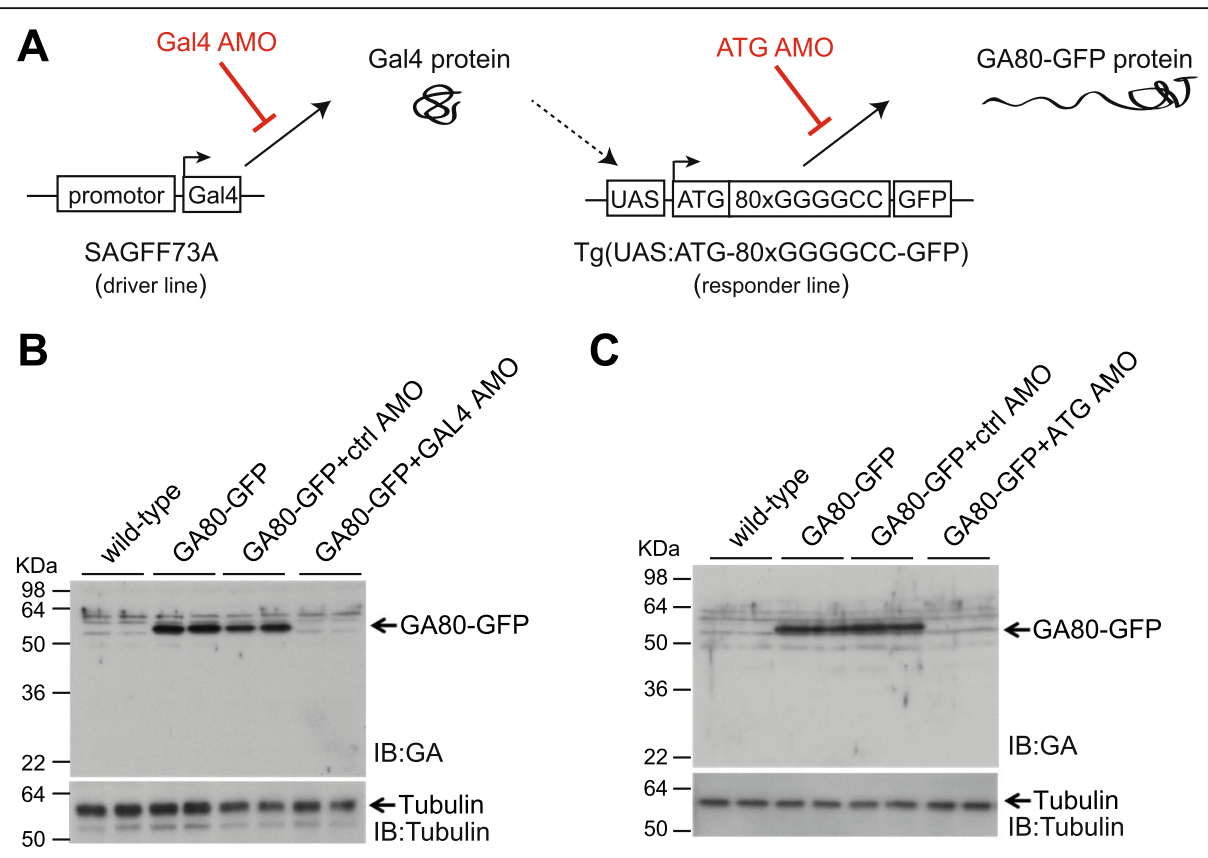

C
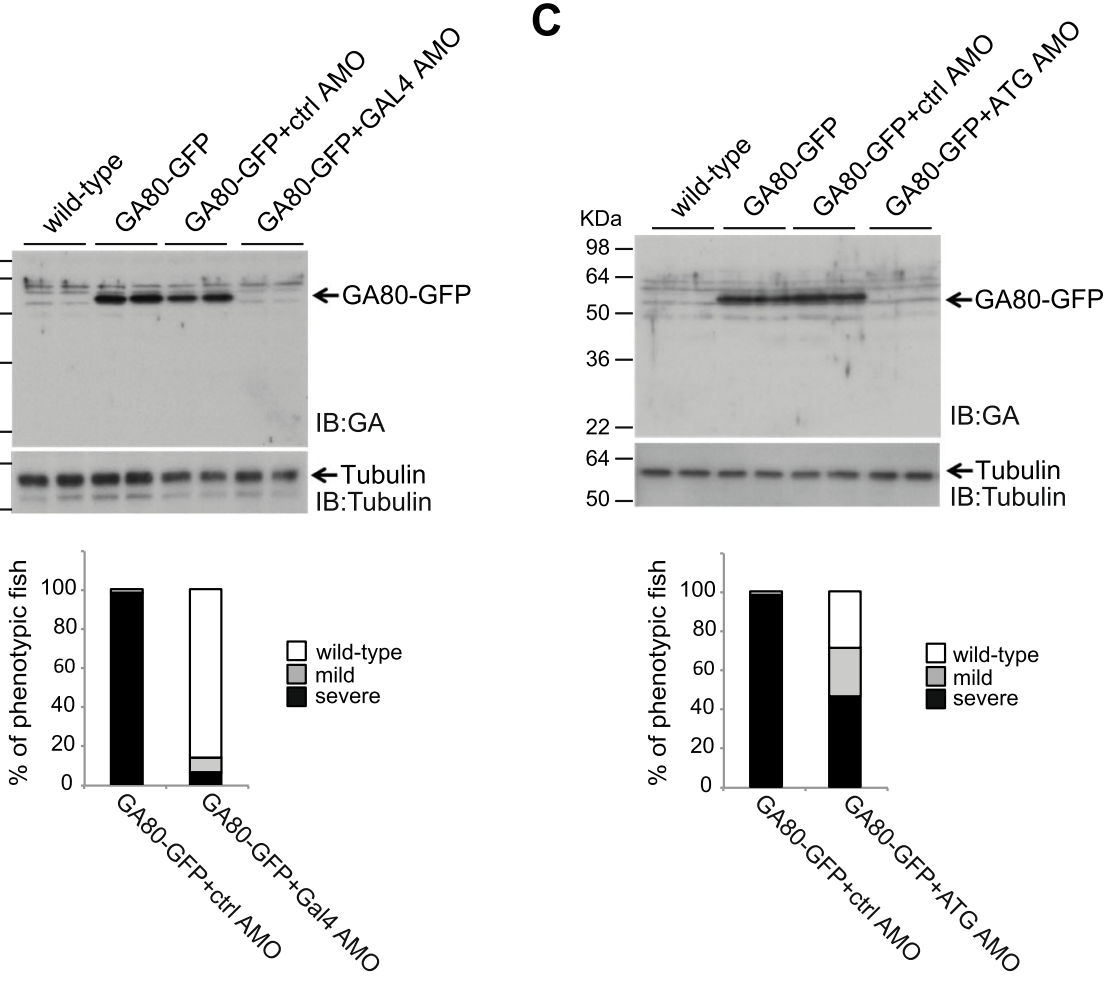

D

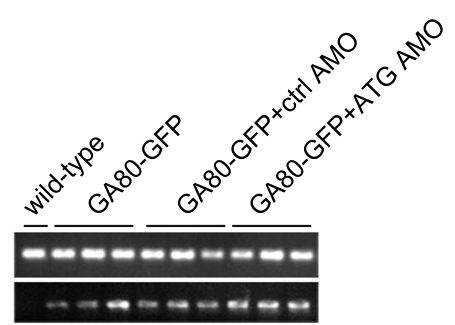

$\mathbf{E}$

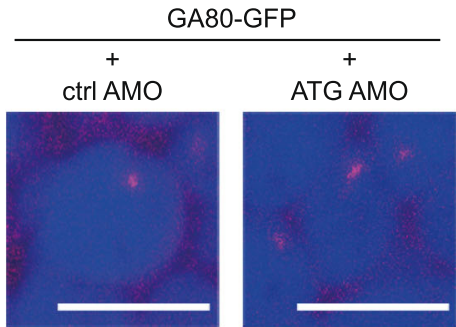

Fig. 4 Antisense morpholino rescued the toxic edema phenotype. a Representation of process of intervention for each morpholino during the generation of GA80-GFP protein in vivo. b GAL4 targeting AMO efficiently blocked Gal4 translation at 2 dpf shown by immunoblotting (upper panel). Quantification of the pericardial edema phenotype observed in GA80-GFP with injection of ctrl AMO or GAL4 targeting AMO at 4 dpf are shown as a bar graph (lower panel) ( $p<0,001,3$ independent experiments with 3 clutches $n \geq 6$ are shown, unpaired $t$ test). c ATG targeting morpholino efficiently inhibited the ATG dependent translation of poly-GA at $2 \mathrm{dpf}$ (upper panel). Quantification of the pericardial edema phenotype observed in GA80-GFP upon injection of ctrl AMO or ATG targeting AMO at $4 \mathrm{dpf}$ shown as a bar graph (lower panel) $(p<0,005,3$ independent experiments with 3 clutches $n \geq 19$ are shown, unpaired $t$ test). $\mathbf{d}$ Semi-quantitative RT-PCR analyses of injected embryos at 2 dpf. e RNA foci formation was not affected upon injection with ctrl AMO or ATG AMO at $2 \mathrm{dpf}$. Scale bar $10 \mu \mathrm{m}$

affecting RNA synthesis [18, 19]. Interestingly, DPRs interfere with nucleocytoplasmic shuttling in Drosophila, cells, and yeast $[21,22,24,25]$. Two independently generated BAC transgenic mouse models recapitulate C9orf72 repeat associated pathology, however they lack neurodegeneration $[34,35]$. In contrast, another BAC transgenic mouse model shows neurodegeneration and TDP-43 pathology [37]. Expression of high levels of C9orf72 repeats by adeno associated virus in mouse brain also generate neurodegeneration and TDP-43 
pathology [20]. These differences might reflect that sufficiently high expression levels are required to induce neurodegeneration. It remains unclear which DPR proteins contribute to ALS/FTLD pathogenesis in patients under physiological conditions. There is currently little evidence for a regional correlation of DPR aggregates in humans and neurodegeneration [38]. These animal models will be valuable tools to further dissect the relative contribution and synergistic effects of repeat RNA and DPRs to toxicity.

GA80-GFP fish showed a circulation defect at $2 \mathrm{dpf}$ and a severe pericardial edema phenotype at $4 \mathrm{dpf}$. Interestingly, double knockout zebrafish (tardbp-/-, tardbpl-/-) also showed circulation defects at $2 \mathrm{dpf}$ and vascular mispatterning, resulting in a pericardial edema phenotype reminiscent of the GGGGCC repeat induced phenotype [39]. Considering that partial loss of TDP-43 function could be linked to the pathogenesis of ALS/ FTLD-TDP-43, including C9orf72 repeat expansion carriers [40], we analyzed expression of Tardbp and Tardbpl_tv1 in GA80-GFP fish. However, no apparent changes in Tardbp and Tardbpl_tv1 protein level were observed upon transgene expression, indicating that neither RNA foci nor poly-GA lead to a loss of TDP-43 function in our zebrafish model. We speculate that potentially a common downstream pathway is affected in double knockout zebrafish (tardbp-/-, tardbpl-/-) and GA80-GFP zebrafish, resulting in a similar circulation defect. However, since pericardial edemas are a common phenotype, which can be caused by a variety of defects, we cannot exclude the possibility that these similar phenotypes have distinct causes. Unfortunately, the larval lethality precludes analysis of possible neurodegenerative phenotypes of the repeat expressing transgenic zebrafish during adulthood.

GA80-GFP leads to inclusion formation which was restricted to the musculature. Why the musculature was more prone to form inclusions in our model remains speculative. This might be due to higher expression levels of the GA80-GFP protein or driven by cell type specific other coaggregating proteins. There was no correlation of cell death and poly-GA aggregation in zebrafish, since we did not observe any degeneration of muscle cells. Interestingly, there is also no clear correlation between DPR aggregate formation and neuronal loss in C9orf72 repeat expansion patients, raising the possibility that the aggregates themselves are not the toxic DPR species $[26,38,41]$.

\section{Conclusion}

We developed a novel vertebrate animal model for C9orf72 repeat expansion pathomechanisms and demonstrated that the DPR poly-GA is toxic in vivo. Selective inhibition of poly-GA production by antisense oligonucleotides decreased toxicity. These findings indicate that intervention with DPR expression might be an effective therapeutic strategy for patients with C9orf72 repeat expansions.

\section{Methods \\ Zebrafish}

Zebrafish embryos were kept at $28.5{ }^{\circ} \mathrm{C}$ and staged as previously described [35]. $\mathrm{AB}$ and TLF were used as the wild-type strains. All experiments were performed in accordance with animal protection standard of Ludwig Maximilians University Munich and approved by the government of upper Bavaria (Regierung von Oberbayern, Munich, Germany).

\section{Antibodies}

Anti-tubulin (Sigma, T6199), anti-GFP (Clontech, 632377), anti-acetylated tubulin (Sigma, T6793), antiTardbp (clone 4A12 [19]), anti-Tardbpl_tv1 (clone 16C8 [19]), anti-znp-1 (DSHB), anti- $\alpha$-actinin (Sigma, A7811), anti-GA (clone 5 F2 [16]), anti-GR (clone 5A2 [9]), antimouse IgG, HRP conj. (Promega, W4021), anti-rabbit IgG, HRP conj. (Promega, W4011), Alexa Fluor antibodies (Invitrogen).

\section{Plasmid construction and generation of transgenic zebrafish}

For the construction of the GA2-GFP and GA80-GFP plasmids, a Kozak sequence (GCCGCCACC) was inserted 3 ' of the ATG.

For the generation of the GA80-GFP and ggggcc80GFP plasmids were constructed as described in Additional file 7: Figure S7. pCS2 + eGFP plasmid [13] was PCR amplified by Phusion high fidelity polymerase (New England Biolabs) using the following primers:

GA80-GFP

A: pCS2-f1: 5' -ggccgcaGGTGGCGGAGGTGGCGTG AGCAAGGGCGAGGAGC-3' pCS2-r1: 5' -gCATGGTGGCGGCCTTGGAT CCGGAATTCGAATCGATGGGATCCTGCA-3'

B: pCS2-f2: 5' - gcaGGTGGCGGAGGTGGCGTGAG CAAGGGCGAGGAGC-3'

pCS2-r2: $5^{\prime}$ - tagCAT

GGTGGCGGCCTTGGATCCGGAATTCGAATCG ATGGGATCCTGCA-3'

ggggcc80xRNA

$A^{\prime}$ : pCS2-f1: 5' - ggccgcaGGTGGCGGAGGTGGCGT GAGCAAGGGCGAGGAGC-3' pCS2-r3: 5' - gGGTGGCGGCCTTGGATCCGGA ATTCGAATCGATGGGATCCTGCA-3'

B' pCS2-f2: 5' - gcaGGTGGCGGAGGTGGC GTGAGCAAGGGCGAGGAGC-3' pCS2-r2: $5^{\prime}$ - tagCATGGTGGCGGCCTTGGATC CGGAATTCGAATCGATGGGATCCTGCA-3' 
After purification of the PCR products generated by $\mathrm{A} / \mathrm{B}$ or A'/B', the PCR products were co-incubated at following cycles $\left(94{ }^{\circ} \mathrm{C} 2 \mathrm{~min}, 94{ }^{\circ} \mathrm{C} 30 \mathrm{~s}, 55^{\circ} \mathrm{C} 30 \mathrm{~s}, 72{ }^{\circ} \mathrm{C}\right.$ $2 \mathrm{~min}, 72{ }^{\circ} \mathrm{C} 10 \mathrm{~min}, 10{ }^{\circ} \mathrm{C} 10 \mathrm{~min}$ ) to produce sticky end fragments digested by NotI/BfaI (New England Biolabs), like for circular plasmid generation. To prepare the gggggcc80-GFP plasmid for the generation of transgenic fish, the pEF-80xGGGGCC plasmid [9] was digested by BamHI and PmeI (New England Biolabs). Subsequently, the $80 x$ GGGGCC fragment was purified and digested by BfaI and NotI. The fragment was then ligated to a PCR amplified pCS2+ plasmid vector backbone.

The pCS2 + ATG-80xGGGGCC-GFP and pCS2 + 80xGGGGCC plasmids were digested by StyI (New England Biolabs) and HpaI (New England Biolabs) and cloned into pT2KXIGdeltaIN plasmid (a gift from K. Kawakami, National Institute of Genetics, Shizuoka, Japan) to generate transgenic zebrafish by TOL2 mediated transposition. Our pT2KXIGdeltaIN plasmid harbored one point mutation in the UAS region that generated a novel StyI recognition site. We therefore had to reintroduce the StyI- StyI digested fragment that was previously lost during the cloning procedure into the pT2 + ATG-80xGGGGCC-GFP/+80xGGGGCC plasmids.

To generate the ATG-2xGGGGCC-GFP plasmid, the primer (ATG-short2:- AAAAGATCCAAGGCCGCCA CCATGCTAGGGGCCGGGGCCGGGGCTCTCAAAC

T-3'), which includes 2xGGGGCC and a T3 primer were used for amplification of the pCS2 + eGFP plasmid backbone [13]. Subsequently, the PCR product as well as pCS2 + ATG-80xGGGGCC-GFP were digested by StyI and HpaI and ligated to each other. The pT2 + ATG2XGGGGCC-GFP plasmid was generated accordingly.

To generate the transgenic fish, $10 \mathrm{ng} / \mu \mathrm{l}$ of the corresponding plasmid and $100 \mathrm{ng} / \mu \mathrm{l}$ transposase mRNA were co-injected into 1 cell stage $\mathrm{AB}$ embryos.

GA2-GFP and GA80-GFP run at a higher molecular weight than the calculated 31,3 and 41,3 $\mathrm{kDa}$ (including the 33aa linker sequences) potentially due to posttranslational modifications. GFP coding sequence without a start codon was used.

\section{Genotyping the repeat length by PCR}

To extract genomic DNA from zebrafish embryos, Proteinase K (Roche) treatment (170 $\mu \mathrm{g} / \mathrm{ml}$, overnight) was performed at $65{ }^{\circ} \mathrm{C}$. Subsequently, Proteinase K was inactivated at $95{ }^{\circ} \mathrm{C}$ for $10 \mathrm{~min}$. To amplify the transgene, GFP specific primers were used for genotyping (GFP-3f: 5' - GTGGTGCCCATCCTGGTCGAGCT-3' and GFP-3r: 5' - AGATCTGAGTCCGGACTTGTAC AG-3').
To confirm the repeat length, Expand Long Template PCR System (Roche) was used with slight modifications: $98^{\circ} \mathrm{C} 10 \mathrm{~min}, 97^{\circ} \mathrm{C} 35 \mathrm{~s}, 55^{\circ} \mathrm{C} 2 \mathrm{~min} 20 \mathrm{~s}, 68^{\circ} \mathrm{C} 2 \min 20 \mathrm{~s}$, $68{ }^{\circ} \mathrm{C} 10 \mathrm{~min}, 55{ }^{\circ} \mathrm{C} 5 \mathrm{~min}, 50{ }^{\circ} \mathrm{C} 5 \mathrm{~min}, 10{ }^{\circ} \mathrm{C}$. Steps from 2 to 4 were repeated in 49 cycles. [36]. 80rep-1f: 5'-CTAGAGGGTATATAATGGATC-3' and 80rep-r2: 5'-CTGTGCTGGATATCTGCAGAATT-3' were used for PCR.

\section{Whole mount fluorescent in situ hybridization and motor axonal length measurement}

Zebrafish embryos (28 hpf) were fixed in $4 \%$ paraformaldehyde (PFA) overnight. Protocol modified from Thisse et al. [42]. The embryo was gradually transferred into phosphate buffered saline Tween-20 (PBST), PBST with 30\% methanol $(\mathrm{MeOH}), \mathrm{PBST}$ with $60 \% \mathrm{MeOH}$ and finally $100 \% \mathrm{MeOH}$ and kept at $-20{ }^{\circ} \mathrm{C}$ overnight. Before Proteinase $\mathrm{K}$ treatment, embryos were transferred into PBST with $60 \% \mathrm{MeOH}$, PBST with $30 \% \mathrm{MeOH}$ and PBST. After Proteinase $\mathrm{K}$ digestion, re-fixation of embryos was performed by PFA for $15 \mathrm{~min}$ at room temperature. After washing with PBST for $5 \times 10 \mathrm{~min}$, embryos were preincubated with hybridization buffer (HYB+) for $1 \mathrm{~h}$ at $65^{\circ}$ C. The Cy3(GGCCCC $)_{4}$ probe was synthesized by IDT (Integrated DNA Technologies) as previously described [2] and diluted into $10 \mathrm{ng} / \mu \mathrm{l}$ in HYB+ solution. Embryos were hybridized overnight at $65{ }^{\circ} \mathrm{C}$. Afterwards, they were washed in HYB- for $3 \times 30 \mathrm{~min}, 2 \times$ saline sodium citrate with $0.1 \%$ Tween 20 (SSCT) for $2 \times 15 \mathrm{~min}, 0.2 \times$ SSCT for $3 \times 30 \mathrm{~min}$, PBST $3 \times 30 \mathrm{~min}$ at $65^{\circ} \mathrm{C}$. After 4,6-diamidin2-phenylindol (DAPI) staining, embryos were mounted in $1.5 \%$ agarose.

DNaseI (Qiagen) or RNaseA (Thermo scientific) treatment of the embryos was performed after Proteinase $\mathrm{K}$ treatment, by incubation with the respective enzyme in PBST for $1.5 \mathrm{~h}$ at $37^{\circ} \mathrm{C}$ prior in situ hybridization.

Measurement of axonal motor neuron axon length was previously described [19].

\section{Semi-quantitative RT-PCR}

The RNeasy kit (Qiagen) was used with on column DNaseI treatment for total RNA isolation. cDNA synthesis was performed with M-MLV reverse transcriptase (Invitrogen) and Random Primer Mix (NEB), followed by a RNaseH (Invitrogen) digest as previously described [16].

\section{Antisense morpholino (AMO)}

Sequences of AMO used in this study:

Control AMO (ctrl AMO) (CCTCTTACCTCAGTTA CAATTTATA), GAL4 targeting AMO (Gal4 AMO) (GTTCGATAGAAGACAGTAGCTTCAT) [37], and ATG targeting AMO (ATG AMO) (CCCCTAGCATGGTGG CGGCCTT) were all obtained from Genetools. AMOs 
were injected into fertilized embryos according to the manufacturer's instructions. The Gal4 AMO and the ATG AMO were used at $125 \mu \mathrm{M}$.

\section{Western blotting and immunohistochemistry}

A standard protocol was used as previously described [16]. To stain the anti- $\alpha$-actinin or anti-acetylated tubulin, in vivo imaging for mCherry expression to analyze the vasculature, embryos at $2 \mathrm{dpf}$ were fixed by $4 \%$ PFA.

\section{Microscopy}

Images were taken with a Cell Observer CSU-X1 (Yokogawa) Spinning Disk (Zeiss), AxioCam MRm (Zeiss) and Evolve 512 (Photometrics) or confocal microscope LSM710 (Zeiss). Brightness and contrast were adjusted using Zen blue or gray (Zeiss) and ImageJ. For in vivo imaging of GFP fluorescence, dechorionated zebrafish were incubated with Tricaine (3-amino benzoic acidethylester) (Sigma) for immobilization. Subsequently, zebrafish were mounted in the Metaphor (low melting temperature) agarose (LONZA).

\section{Additional files}

Additional file 1: Figure S1. RNA foci formation overview. Embryos of the indicated genotypes stained with a Cy3-labeled probe to visualize RNA foci formation by in situ hybridization. Between 13-33 cells per field of view showed RNA foci in the GA80-GFP larvae. All images were taken without DAPI fluorescence. Scale bar $10 \mu \mathrm{m}$. (PDF $2702 \mathrm{~kb}$ )

Additional file 2: Figure S2. Spinal motor neuron axonal outgrowth is not affected. (A) Spinal motor neuron axon of GA80-GFP fish (Gal4 driver + UAS:ATG-80xGGGGCC-GFP responder) and the GFP negative siblings (Gal4 driver or UAS:ATG-80xGGGGCC-GFP alone, or wild-type) at $28 \mathrm{hpf}$. (B) Length of outgrowing spinal motor neuron axons measured from the exit point of the spinal cord to the tip of the growth cone in the 5 somites anterior of the end of the yolk expansion at $28 \mathrm{hpf}$ (indicated by the numbers 1-5). Embryos are sorted by the genotypes wild-type, driver, responder, and driver + responder. Statistical analyses was performed in indicated genotypes. Scale bar $20 \mu \mathrm{m}$. Mean \pm SD. (PDF $4905 \mathrm{~kb}$ )

Additional file 3: Figure S3. Overall neuronal outgrowth is not affected. Overall neuronal outgrowth was analyzed in embryos stained with an antibody against acetylated tubulin at $2 \mathrm{dpf}$. Siblings of GA80GFPa zebrafish expressing GFP (A) or not expressing GFP (B). Scale bar 100 нm. (PDF 6302 kb)

Additional file 4: Figure S4. Muscle patterning is not affected. (A) Quantification of GFP inclusions in GA80-GFPa and GA80-GFPb larvae subdivided into mild and strong edema phenotypes at $4 \mathrm{dpf}$. ( $n=4$ per subgroup, mean \pm SD). Amount of inclusions from GA80-GFP line $a$ and $b$ with mild and strong phenotypes were not significantly different (paired t-test). Inclusions were exclusively detected in the musculature in both lines. (B) The overall structure of the muscle was analyzed by a-actinin staining at $2 \mathrm{dpf}$ in a GFP negative GA80-GFP embryo and (B) GFP positive sibling. Scale bar $20 \mu \mathrm{m}$. (PDF $3472 \mathrm{~kb}$ )

Additional file 5: Figure S5. Vascular patterning is not affected. The vasculature was analyzed by incrossing with $\mathrm{Tg}(\mathrm{kdrl}: \mathrm{HsHRAS}-\mathrm{mCherry})^{5896}$ into the GA80-GFP expressing lines. mCherry expressed from the $\mathrm{Tg}(\mathrm{kdrl}: H s H R A S-m C h e r r y)^{5896}$ transgene is shown in Ga80-GFP-a transgenic zebrafish not expressing GFP (A) and siblings expressing GFP (B) at $2.5 \mathrm{dpf}$. Scale bar 20 нm. (PDF 4003 kb)

Additional file 6: Figure S6. Tardbp function is not impaired in repeat expressing fish. (A) GA80-GFPa zebrafish expressing GFP and (B) siblings not expressing GFP. Western blot analysis of $2 \mathrm{dpf}$ old embryos with antibodies as indicated. Tardbp/Tardbpl_tv1 bands indicated by arrow heads. (PDF $3086 \mathrm{~kb}$ )

Additional file 7: Figure S7. Construction of repeat expressing plasmid in zebrafish. A representative scheme to generate the $80 x g g g g c c$ repeat containing plasmid. (PDF $1457 \mathrm{~kb}$ )

\section{Acknowledgements}

We thank B. Solchenberger, F. van Bebber, K. Strecker, L. Hasenkamp and S. Rothhaemel for the helpful discussion. K. Strecker for taking images by confocal microscopy. E. Kremmer for generating antibodies. W. Katharina, A. Rechenberg and M. Graf for technical assistance. S. Schlink and R. Rojas Rojas for taking care of zebrafish. This work was supported by the Helmholtz cross-program topic "Metabolic Dysfunction" to B. S., the Uehara Memorial Foundation and the National BioResource Project from the Ministry of Education, Culture, Sports, Science and Technology of Japan to K.K. and the European Research Council under the European Union's Seventh Framework Programme FP7/2014-2019 under grant agreement nº 617198 [DPR-MODELS] to D.E..

\section{Authors' contributions}

$\mathrm{YO}, \mathrm{AH}$, and $\mathrm{BS}$ designed the research. $\mathrm{KA}, \mathrm{KK}$, and DE shared materials. $\mathrm{YO}$, $\mathrm{AW}-\mathrm{W}$, and $\mathrm{AH}$ performed the experiments. $\mathrm{YO}, \mathrm{KA}, \mathrm{KK}, \mathrm{CH}, \mathrm{DE}$ and $\mathrm{BS}$ wrote the paper. All authors read and approved the final manuscript.

\section{Competing interests}

The authors declare that they have no competing interests.

\section{Author details}

'German Center for Neurodegenerative Diseases (DZNE),

Feodor-Lynen-Str.17, 81377 Munich, Germany. ${ }^{2}$ Biomedical Center, Biochemistry, Ludwig-Maximilians University Munich, Feodor-Lynen-Str.17, 81377 Munich, Germany. ${ }^{3}$ Division of Molecular and Developmental Biology, National Institute of Genetics, Mishima, Shizuoka 411-8540, Japan. ${ }^{4}$ Munich Cluster for Systems Neurology (SyNergy), Feodor-Lynen-Str.17, 81377 Munich, Germany.

Received: 17 September 2015 Accepted: 24 December 2016 Published online: 14 January 2017

\section{References}

1. Gijselinck I, et al. A C9orf72 promoter repeat expansion in a Flanders-Belgian cohort with disorders of the frontotemporal lobar degenerationamyotrophic lateral sclerosis spectrum: a gene identification study. Lancet Neurol. 2012;11(1):54-65.

2. Renton $A E$, et al. A hexanucleotide repeat expansion in C9ORF72 is the cause of chromosome 9p21-linked ALS-FTD. Neuron. 2011;72(2):257-68.

3. DeJesus-Hernandez M, et al. Expanded GGGGCC hexanucleotide repeat in noncoding region of C9ORF72 causes chromosome 9p-linked FTD and ALS. Neuron. 2011:72(2):245-56.

4. Majounie $\mathrm{E}$, et al. Frequency of the C9orf72 hexanucleotide repeat expansion in patients with amyotrophic lateral sclerosis and frontotemporal dementia: a cross-sectional study. Lancet Neurol. 2012;11(4):323-30.

5. Renton AE, Chio A, Traynor BJ. State of play in amyotrophic lateral sclerosis genetics. Nat Neurosci. 2014;17(1):17-23.

6. Lagier-Tourenne C, et al. Targeted degradation of sense and antisense C9orf72 RNA foci as therapy for ALS and frontotemporal degeneration. Proc Natl Acad Sci U S A. 2013;110(47):E4530-9.

7. Haeusler AR, et al. C9orf72 nucleotide repeat structures initiate molecular cascades of disease. Nature. 2014;507(7491):195-200.

8. Mori K, et al. hnRNP A3 binds to GGGGCC repeats and is a constituent of p62-positive/TDP43-negative inclusions in the hippocampus of patients with C9orf72 mutations. Acta Neuropathol. 2013;125(3):413-23.

9. Lee $Y B$, et al. Hexanucleotide repeats in ALS/FTD form length-dependent RNA foci, sequester RNA binding proteins, and are neurotoxic. Cell Rep. 2013:5(5):1178-86

10. Todd PK, et al. CGG repeat-associated translation mediates neurodegeneration in fragile $X$ tremor ataxia syndrome. Neuron. 2013;78(3): 440-55. 
11. Zu T, et al. Non-ATG-initiated translation directed by microsatellite expansions. Proc Natl Acad Sci U S A. 2011;108(1):260-5.

12. Ash PE, et al. Unconventional translation of C9ORF72 GGGGCC expansion generates insoluble polypeptides specific to C9FTD/ALS. Neuron. 2013;77(4): 639-46

13. Mori K, et al. The C9orf72 GGGGCC repeat is translated into aggregating dipeptide-repeat proteins in FTLD/ALS. Science. 2013;339(6125):1335-8.

14. Mori $\mathrm{K}$, et al. Bidirectional transcripts of the expanded C9orf72 hexanucleotide repeat are translated into aggregating dipeptide repeat proteins. Acta Neuropathol. 2013;126(6):881-93.

15. Zu T, et al. RAN proteins and RNA foci from antisense transcripts in C9ORF72 ALS and frontotemporal dementia. Proc Natl Acad Sci U S A. 2013;110(51):E4968-77.

16. May S, et al. C9orf72 FTLD/ALS-associated Gly-Ala dipeptide repeat proteins cause neuronal toxicity and Unc119 sequestration. Acta Neuropathol. 2014;128(4):485-503.

17. Zhang YJ, et al. Aggregation-prone c9FTD/ALS poly (GA) RAN-translated proteins cause neurotoxicity by inducing ER stress. Acta Neuropathol. 2014;128(4):505-24.

18. Mizielinska S, et al. C9orf72 repeat expansions cause neurodegeneration in drosophila through arginine-rich proteins. Science. 2014:345(6201):1192-4.

19. Kwon I, et al. Poly-dipeptides encoded by the C9orf72 repeats bind nucleoli, impede RNA biogenesis, and kill cells. Science. 2014;345(6201):1139-45.

20. Chew J, et al. Neurodegeneration. C9ORF72 repeat expansions in mice cause TDP-43 pathology, neuronal loss, and behavioral deficits. Science. 2015;348(6239):1151-4.

21. Freibaum BD, et al. GGGGCC repeat expansion in C9orf72 compromises nucleocytoplasmic transport. Nature. 2015;525(7567):129-33.

22. Zhang $\mathrm{K}$, et al. The C9orf72 repeat expansion disrupts nucleocytoplasmic transport. Nature. 2015;525(7567):56-61.

23. Zhang $Y$ J, et al. C9ORF72 poly (GA) aggregates sequester and impair HR23 and nucleocytoplasmic transport proteins. Nat Neurosci. 2016;19(5):668-77.

24. Jovicic A, et al. Modifiers of C9orf72 dipeptide repeat toxicity connect nucleocytoplasmic transport defects to FTD/ALS. Nat Neurosci. 2015;18(9):1226-9.

25. Boeynaems $S$, et al. Drosophila screen connects nuclear transport genes to DPR pathology in c9ALS/FTD. Sci Rep. 2016;6:20877.

26. Schludi $\mathrm{MH}$, et al. Distribution of dipeptide repeat proteins in cellular models and C9orf72 mutation cases suggests link to transcriptional silencing. Acta Neuropathol. 2015;130:537-55.

27. Kawakami K. Tol2: a versatile gene transfer vector in vertebrates. Genome Biol. 2007:8 Suppl 1:S7.

28. Asakawa K, Kawakami K. The Tol2-mediated Gal4-UAS method for gene and enhancer trapping in zebrafish. Methods. 2009;49(3):275-81.

29. Anonymous (!!! INVALID CITATION !!! \{Zhang, 2014 \#17;Mizielinska, 2014 \#18; Kwon, 2014 \#28;Chew, 2015 \#44;Zhang, 2014 \#17\}).

30. Gendron TF, et al. Antisense transcripts of the expanded C9ORF72 hexanucleotide repeat form nuclear RNA foci and undergo repeat-associated non-ATG translation in C9FTD/ALS. Acta Neuropathol. 2013;126(6):829-44.

31. Haeusler AR, Donnelly CJ, Rothstein JD. The expanding biology of the C9orf72 nucleotide repeat expansion in neurodegenerative disease. Nat Rev Neurosci. 2016;17(6):383-95.

32. Mori $K$, et al. Reduced hnRNPA3 increases C9orf72 repeat RNA levels and dipeptide-repeat protein deposition. EMBO Rep. 2016;17(9):1314-25.

33. Hukema RK, et al. A new inducible transgenic mouse model for C9orf72associated GGGGCC repeat expansion supports a gain-of-function mechanism in C9orf72-associated ALS and FTD. Acta Neuropatho Commun. 2014;2:166.

34. O'Rourke JG, et al. C9orf72 BAC transgenic mice display typical pathologic features of ALS/FTD. Neuron. 2015;88(5):892-901.

35. Peters OM, et al. Human C9ORF72 hexanucleotide expansion reproduces RNA foci and dipeptide repeat proteins but not neurodegeneration in BAC transgenic mice. Neuron. 2015:88(5):902-9.

36. Wen X, et al. Antisense proline-arginine RAN dipeptides linked to C9ORF72ALS/FTD form toxic nuclear aggregates that initiate in vitro and in vivo neuronal death. Neuron. 2014;84(6):1213-25.

37. Liu Y, et al. C9orf72 BAC mouse model with motor deficits and neurodegenerative features of ALS/FTD. Neuron. 2016;90(3):521-34.

38. Mackenzie IR, et al. Dipeptide repeat protein pathology in C9ORF72 mutation cases: clinico-pathological correlations. Acta Neuropathol. 2013;126(6):859-79.
39. Schmid B, et al. Loss of ALS-associated TDP-43 in zebrafish causes muscle degeneration, vascular dysfunction, and reduced motor neuron axon outgrowth. Proc Natl Acad Sci U S A. 2013;110(13):4986-91.

40. Lee EB, Lee VM, Trojanowski JQ. Gains or losses: molecular mechanisms of TDP43-mediated neurodegeneration. Nat Rev Neurosci. 2012;13(1):38-50.

41. Gomez-Deza J, et al. Dipeptide repeat protein inclusions are rare in the spinal cord and almost absent from motor neurons in C90RF72 mutant amyotrophic lateral sclerosis and are unlikely to cause their degeneration. Acta Neuropathol Commun. 2015;3(1):38.

42. Thisse C, Thisse B. High-resolution in situ hybridization to whole-mount zebrafish embryos. Nat Protoc. 2008:3(1):59-69.

\section{Submit your next manuscript to BioMed Central and we will help you at every step:}

- We accept pre-submission inquiries

- Our selector tool helps you to find the most relevant journal

- We provide round the clock customer support

- Convenient online submission

- Thorough peer review

- Inclusion in PubMed and all major indexing services

- Maximum visibility for your research

Submit your manuscript at www.biomedcentral.com/submit 